\title{
Test of Strain Behavior Model with Radon Anomaly in Seismogenic Area: A Bayesian Melding Approach
}

\author{
Pushan Kumar Dutta ${ }^{1,2}$, Mrinal Kanti Naskar ${ }^{1,2}$, O. P. Mishra ${ }^{3,4}$ \\ ${ }^{1}$ Advanced Digital and Embedded System Lab, Electronics and Tele-Communication Department, Jadavpur University, Kolkata, India \\ ${ }^{2}$ Electronics and Tele-Communication Department, Jadavpur University, Kolkata, India \\ ${ }^{3}$ SAARC Disaster Management Centre (SDMC), New Delhi, India \\ ${ }^{4}$ Geological Survey of India, Kolkata, India \\ Email: \{ascendent1,opmishra2010.saarc\}@gmail.com, mrinalnaskar@yahoo.co.in
}

Received August 18, 2011; revised September 17, 2011; accepted October 25, 2011

\begin{abstract}
Mathematical models in seismo-geochemical monitoring offer powerful tools for the study and exploration of complex dynamics associated with discharge of radon as the indicator of change of intense - deformed conditions of seismogenic layers or blocks within the lithosphere. Seismic precursory model of radon gas emanation in the process of earthquake prediction research aims to find out the distinct anomaly variation necessary to correlate radon gas with processes of preparation and realization of tectonic earthquakes in long-term and short-term forecasts tectonic earthquakes. The study involves a radon gas volume analytic model to find the correlation of radon fluctuations to stress drop under compression and dilatation strain condition. Here, we present a mathematical inference by observing radon gas emanation prior to the occurrence of earthquake that may reduce the uncertainties in models and updating their probability distributions in a Bayesian deterministic model. Using Bayesian melding theorem, we implement an inferential framework to understand the process of preparation of tectonic earthquake and concurrent occurrence of radon discharge during a tectonic earthquake phenomena. Bayesian melding for deterministic simulation models was augmented to make use of prior knowledge on correlations between model inputs. The background porosity is used as a priori information for analyzing the block subjected to inelastic strain. It can be inferred that use of probabilistic framework involving exhalation of radon may provide a scenario of earthquake occurrences on recession of the curve that represents a qualitative pattern of radon activity concentration drop, indicating associated stress change within the causative seismogenic fault. Using evidence analysis, we propose a joint conditional probability framework model simulation to understand how a single fracture may be affected in response to an external load and radon anomaly change that can be used to detect the slip, a predictable nature of the causative fault in the subsurface rock.
\end{abstract}

Keywords: Radon; Deterministic Model; Probability Distribution; Strain, Bayesian Melding; Seismogenic Layer; Earthquake Prediction

\section{Introduction}

Geochemical and hydrological phenomena has attracted much attention in the study of the process of earthquake prediction studies to detect anomalous change in the concentration of subsurface gas prior to an earthquake. There are many published records and evaluation studies on geochemical precursors reporting the detection of validated precursory phenomenon between geochemical data and seismic activity [1]. Anomalous change in subsurface soil gas radon concentration have been observed to precede earthquake occurrence and therefore radon has potential use in earthquake prediction studies [2]. The first evidence of a precursory correlation between radon and earthquake occurrence came from observation of radon concentration in well water prior to the Tashkent earth- quake of 1966 [3].The radon gets effected by opening or closing of cracks resulting releasing or confining the gas in deep earth. Crustal stress change creates new solid-fluid interfaces resulting in more than one pathways for the emanation of radon. Earthquake occurs in three phases of quasi elastic strain of compression and dilatation phenomena where strain decrease or increase has a subsequent impact on the radon activity. The process of earthquake occurrence is always accompanied by strain deformations resulting in the phenomena of complex short- or long-term anomalous variations [4] of radon concentration in groundwater and soil gases prior to earthquakes reported from various regions of the world [5]. It was observed by [6] a radon anomaly which coincided with several other geophysical and geochemical anomalies and appeared to have been associated with an earthquake. The 
occurrence of the anomaly of the related sub-surface gases provides strong support of the long range effects of the occurrence of large earthquakes in the intra-plate region and the occurrence of regional scale strain events prior to occurrence of larger earthquakes. The existing geophysical models are elastic soft inclusion model [7] and the dislocation model by [8] have found significant impact between the observed geo-chemical anomalies to find the buildup of stress having significant impact on the strain deformation within the crust. However, no significant research has been done so far to establish a probabilistic framework to explain change of concentration of radon and occurrence of other geochemical phenomena before earthquake event. According to dilatation model in a block for earthquake occurrence [9] when regional stress increases, dilation of rock masses could cause a change in the surface area of rocks matrix (Figure 1) due to cracking, or in the flow rate of pore fluids as they are forced out of the interstitial space. Both of these processes will enhance the transport of radon from its original enclosures into the ground water. The major work involves the study of the background seismicity and also the anomalous radon fluctuation involved in the study of earthquake [10] which has been used in our study by the deterministic simulation model. One of the contributions in this paper is an assessment of radon emanation model [11] for radon volume analysis to assess radon concentration in a deterministic model of natural geodynamic processes which motivates our use of a bayesian framework termed bayesian melding [12] for estimating model parameters and model outputs. Bayesian melding yields probabilistic predictions for various quantities of interest, behavior of radon under strain conditions and relationship of pore pressure data of rock for the dynamics of radon exhalation prior to tectonic earthquakes clearly following a spatial zonation pattern. This kind of modeling

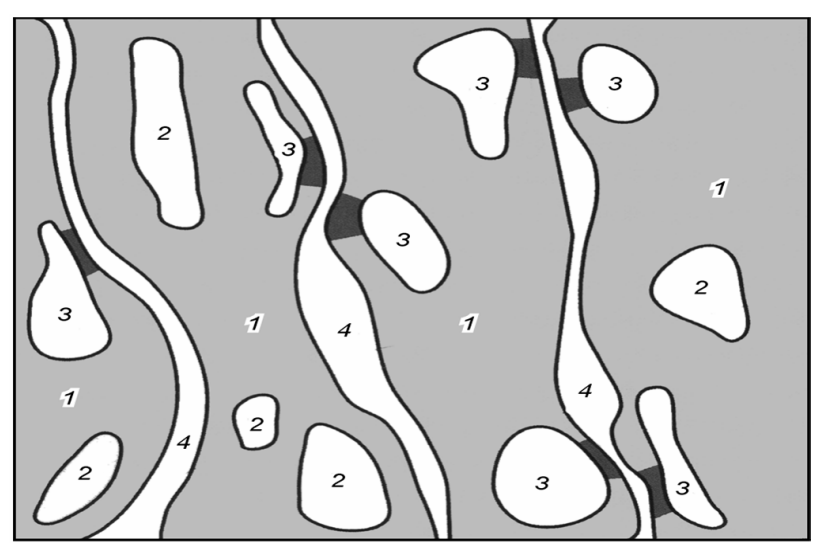

Figure 1. Suggested pore-crack model. 1, rock matrix; 2, isolated pores; 3 , pores degrading under stress change; 4 , cracks. Dark color marks rock under failure. (V. I. Utkin and A. K. Yurkov/Russian Geology and Geophysics 51 (2010) 220-227 221 pg. 1 Figure 1). to draw bayesian inference with pooled prior and likelyhood ratio is called melding. The word melding is used as a collective word for all quantitative knowledge related to the input strain deformation, radon anomaly and subsequent stress drop used in proposed work for the first time to provide a full assessment of associated uncertainty in a tectonic earthquake process. The study is categorized keeping in mind radon as a seismic precursor. In Section 2, radon has been used for an elaborate study based on its behavior of radon with change of porosity and deformation. In Section 3, we give background of bayesian deterministic simulation model for stress drop and illustrate the method using a dynamics model through a step by step parameter estimation approach. In Section 4, we discuss limitations of and possible improvements to our approach of study.

\section{Diagnosis of Radon Volume Analysis (RVA) in Tectonic Earthquake}

The main objective of this study is to establish a bayesian framework for parameter estimation in the nature of variation of radon concentration in soil gas as a possible precursor to earthquakes in the Himalayan belt. Earthquakes prepare due to a pseudo-elastic condition of the top lithospheric blocks. The quasi-elastic surrounding medium with a rock mass is able to transmit elastic stresses through the preparation region but possesses inelastic properties giving rise to surface precursors or anomalous disturbances. It is known that lithospheric block in critical stress condition is likely to undergo rupture and release radon and similar geochemical gases in this condition. The increase in soil-gas radon concentration before an earthquake may be due to the strain buildup in the area. The dilation of brittle rock mass occurs at a rate faster than the recharge of pore water and gas saturation developed in newly created cracks preceding the above mentioned earthquakes. During this process, very small fractures are formed in the rocks, which help to contribute more radon to the soil-gas near the Earth's surface. A decreasing radon anomaly may be due to the squeezing effect of compressional stress in rock, which changes the porosity of soil at a micro-scale [13]. These models for measurement of uncertainty, considered in a Bayesian conditional independence framework generated from the study of previously recorded data. Elastic strain eventually causes rocks to dilate (increase in volume) when stress on rocks $>50 \%$ of the rock strength opening fractures develop with minor seismicity [14]. The rate and extent of radon emission can be well correlated with geodetic strain prior to occurrence of a particular earthquake. Radon data is highly correlated with in-situ geodetic strain, stress drop and the nature of sub-surface rock materials to adjudge a suitable precursor for earthquake forecast under earthquake prediction research. 
The regional scale nature of precursory deformations can help us understanding the local dynamics of the subsoil radon field controlled by variations in the stress-strain state in tectonically un compacted regions of the crust was proposed in $[15,16]$. In this paper, we derive a probabilistic bayesian framework between emanation fields and the dynamics related to the vibro-seismic effect within the rock matrix that can lead us to the study of the time of possible catastrophic event based on radon correlation matrix (Figure 1) breakage. For rock friction the stress released will be dependent on the matrix condition and reflected immediately as change of pore pressure. Any rock fits a model of a medium consisting of a matrix with randomly distributed open cracks and closed pores. Radon gas in rocks partly remains in the solid matrix and partly moves to pore fluids where it migrates through interconnected pores, fissures by diffusion and fluid flow [17]. This process of seismo-geochemical emanation from the earth crust is not uniform in spaces and also is controlled by the distribution of stress conditions of fractures in crust. Crustal stress drop creates new solid-fluid interfaces causing more emanation of radon. [18] and [19] showed an increase in radon emanation associated with micro-cracking and changes in volumetric strain by conducting uni-axial experiments. The emanation is associated with transient crustal deformation [20] based on observations of a tunnel in the vicinity of the two lakes. Radon emanation from the close pore matrix exists partly in the space of the closed pores, partly in the cracks, and some is adsorbed by the free inner surface. These emissions are considered as precursors of general fracture. Radon is always let out depending on the permeability of the soil which is the characteristic of the pores present in the medium. Exhalation of radon from a lithospheric block is key parameter defining change of permeability of environment. Radon and change of the intense-deformed condition of environment is key to the study of the process of slip nucleation. Radon monitoring has a time span of 100 days which is the time of last stage of preparation of earthquake as determined by [20]. The behavior of radon exhalation reflects properties of environment and the strain deformation nature with respect to porosity. Porosity in the volume fraction of interstitial void spaces comprising of pores, cracks along various seismic and geodetic environments responsible for radon migration and rock fracture and time invariant strain deformation [21]. The decrease (increase) reaches significant values approximately 90 - 100 days prior to an event. Behavior of radon in the conditions of stress change for radon activity concentration per unit volume in soil gas at the initial stress $\mathrm{Q} 0$ corresponds to the radon concentration $\mathrm{C} 0$ in a rock (Figure 2).

Under compression radon increases and becomes greater than Q0), radon first rises as the volume of cracks

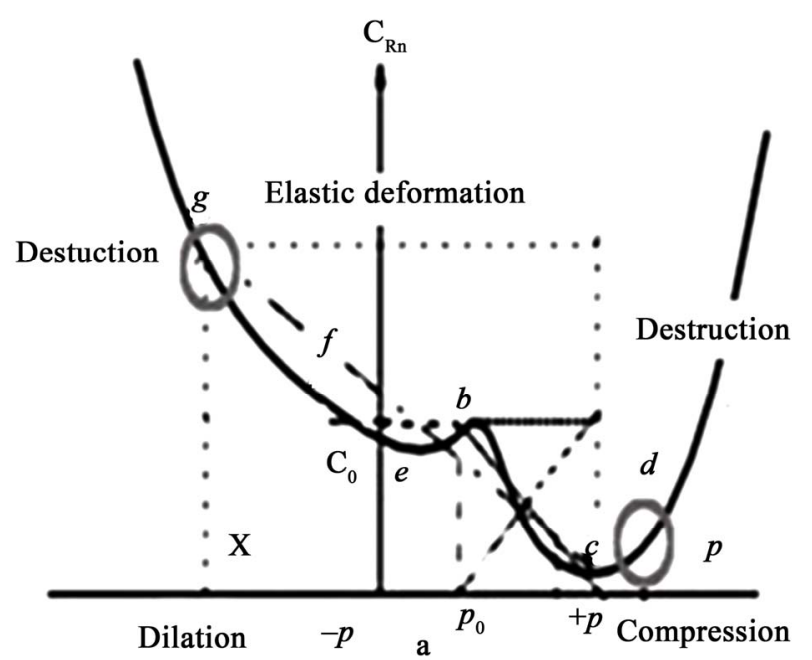

Figure 2. Radon activity concentration $\left(C_{R n}\right)$ changes associated with stress change (Q) (Utkin \& Yurokov, 2010 pg 309 Figure 2).

contracts, and then it falls after the cracks close. Later on, as the stress grows, rocks become subject to failure, weak links between pores break down, and radon activity increases notably. Under extension ( $Q$ decreases and becomes less than Q0), radon first falls as the volume of cracks expands, but then it rises after the cracks broaden and the pores open. Thus, there exists some domain of quasi-elastic strain in which compression and extension strains decrease or increase the radon activity respectively. Initial increase in pressure from point $\mathrm{P}_{\mathrm{o}}$ which is the initial stress condition (a) leads all over again to small increase in concentration of radon (effect of pumping, a point $b$ ), then reduction pores spaces occurs - concentration decreases (point c), then the basis of breed (times incorporate and there is an emission of radon, a (point $\mathrm{d}$ ) collapses. At a dilatation stage of a block (reduction $\mathrm{P}_{\mathrm{o}}$ ) times all over again increase (concentration decreases, a (point e), then new channels open (concentration increases - a point $f$ ), then the file collapses also emission of radon occurs (a point $\mathrm{g}$ ). The behavior of radon describes this process unequivocally enough. Processes in various environments differ among themselves only in size of initial concentration of radon. Between points a and $g$ it is possible to consider a parity linear association for a case of approximation in a faltering line. All processes from a point a up to a point $\mathrm{c}$ (or from a point a up to a point $\mathrm{g}$ ) have duration of 90 - 100 days. At deformation of an earth's crust before earthquake, there are zones of compression and a stretching of a rock matrix subject to strain conditions. It means, that the monitor of radon will show either reduction of radon, or increase in radon. All depends on that is in what zone detector gauge is located. We use the evidence of precursory phenomena of soil gas anomaly and sudden drop prior to an earthquake to eva- 
luate the posterior odds of strain deformation increase. If a lithospherical block is not in a critical condition or is under pressure any trigger's function cannot cause earthquake. The lithospheric block should save up energy that could lead to a discharge of a pressure of a block. Thus the stronger external influence, the greater energy dump and the more strong aseismic faulting takes place. Inductive probability approach has been implemented in understanding radon anomaly decline before large earthquake. From local, regional, or global perspectives, one of the scientific conditions to our understanding is of the extreme event of reaching strain deformation condition and the corresponding likelihood of its occurrence.

\section{Diagnosis of Radon Volume Data Analysis}

The prior odds and likelihood described above are combined to produce the posterior distribution that is used to make inference about the parameters, as well as probabilistic forecasts through a method of study of experimental or field data to reduce the uncertainties in models, by updating those probability distributions. Proper handling of such uncertainties is key to the successful usage of models to predict experimental or field observations. This problem has been addressed over the years by many tools for model calibration and parameter estimation. In this article we present a general framework for uncertainty analysis and parameter estimation that is designed to handle uncertainties associated with the modeling of dynamic models.

\subsection{Bayesian Melding}

A growing theme in mathematical modeling of environmental study is uncertainty analysis. Bayesian melding, a method for assessing uncertainties in deterministic simulation models, was augmented to make use of prior knowledge about correlations between model inputs. The melding module provides a bayesian framework to analyze uncertainty in mathematical models. The method was developed initially for deterministic simulation models and can be applied to crack propogation model in homogenous hierarchial medium with uniform stress when the strain deformation reaches the maximum value [22] includes tools that allow modelers to integrate prior information about the model's parameters and variables into the model, in order to explore the full uncertainty associated with a model. The posterior distributions are estimated by sampling-importance-resampling [23]. We apply a posterior density that is proportional to the prior density times the likelihood. Bayesian melding is a way of putting the analysis of simulation models on a solid statisticcal basis. In such cases, it may be interesting to use this information, to estimate the parameter values which maximize the fit of our model to the data, number of samples we will take from the joint prior distribution of the parameters to run the inference. The basic idea is to combine all the available evidence about model inputs and model outputs in a coherent bayesian way to yield a bayesian posterior distribution of the quantities of interest. We have $\mathrm{H}_{1}$, which is our hypothesis that a strain deformation increases with radon anomaly change $\mathrm{G}$ with porosity and rupture breakage $\mathrm{E}$ being our evidence. The analysis for this paper consists of three parts, involves replicating radon variation analysis; estimates the parameters and comparing them to the estimates obtained via Bayesian melding (BM) with the help of data (model selection) and estimate data which cannot be directly observed, with the help of theory for arguments (parameter estimation). Bayesian arguments give an expectation value of radon anomaly change with pore pressure when a certain lithospherical block undergoes deformation of $\mathrm{N}$ number of blocks. This framework help us realize the expectation as how any two blocks can undergo slip patch with the variation in the radon anomaly drop prior to any earthquake. However the pore precipitation, the spatial distribution of the generation rate of the background evidence is difficult to perceive and deviates from one surrounding to another.

\subsection{Algorithm}

Defining the models parameters and initial conditions, and a function which takes in the parameters runs the model and returns the output. We have assimilated a suitable algorithm for radon volume data analyses to detect the slip, a predictive nature of the causative fault in the sub-surface rock.

1) A random sample of size $M$ from the values of 0 from its prior distribution $\mathrm{q}_{\theta}(\theta)$. denote the sample by $\left(0_{1}, 0_{2}, \cdots, 0_{\mathrm{M}}\right)$.

2) Estimating finite mixtures by determining the number of terms or component densities $\mathrm{c}$ in the mixture through an initial guess at the component parameters mixing coefficients, means, and covariance matrices for each multivariate normal density.

3) Calculate the posterior probability and the current values of the parameters as the 1-Step.

4) Update the mixing coefficients, the means, and the covariance matrices for the individual components. This is the 2-Step.

5) Continue the iteration until the changes in the estimates at each iteration are less than some pre-set tolerance.

6) Keep iterating until the likelihood function converges.

7) Instantiate our fitting Object.

8) Instantiate few arguments: the first n number of samples we will take from the joint prior distribution of the parameters to run the inference. The second one (model) is the callable (function) which corresponds to the model you want to fit to data. 
9) Find the specification of the prior distributions for all parameters included in the model need to be included in the analysis.

10) Finding recursive bayesian fitting function having posterior outlook generated in smaller window for fitting the inferential framework.

11) Find the marginal posterior distribution provided that conditional distribution of $\theta$ given both $\mathrm{D}$ and model $\mathrm{M}$ to partition $\mathrm{D}$ into components for an inferential model.

\subsection{Model's Inputs and Outputs to Radon Volume Analysis for an Inferential Framework}

Let $\mathrm{E}$ denote one or more items of evidence of porosity of rocks in totality. We need to consider how this evidence affects the hypothesis $\mathrm{H}_{1} \cdot \mathrm{H}_{1}$ the condition that deformation strain increase cause earthquake. The probability of the increase in strain deformation results in rise of radon anomaly is established. Increase of radon anomaly occurs as dilatation zones are created and micro pores change configuration. Prior probabilities of micro fractures will be updated with new information to create posterior probabilities as to when deformations exceed the critical condition to create rupture. Thus in this case of strain rise with a single precursory increase against a single volumetric strain rise, the evidence presented is that the blocks porosity and subsequent fault creep matches the region predictive hypothesis, $\mathrm{H}_{1}$, is that of radon anomaly variation $(\mathrm{G})$ occurs for the region. One assess the conditional probability for either hypothesis, given the evidence $\mathrm{E} \operatorname{Pr}\left(\mathrm{H}_{1} \mid \mathrm{E}\right)$ that is how porosity effects the deformation strain. The left-hand side of (1) is the posterior odds $\mathrm{P}\left(\mathrm{G}, \mathrm{H}_{1}, \mathrm{E}\right)$ is the probability that radon anomaly occurs with deformation strain under the background evidence of pore pressure. $\mathrm{P}\left(\mathrm{H}_{1} / \mathrm{G}, \mathrm{E}\right)$ is the condition that maximum deformation occurs with radon anomaly change and increasing porosity.

$$
\begin{aligned}
& \mathrm{P}\left(\mathrm{H}_{1} / \mathrm{G}, \mathrm{E}\right)= \mathrm{P}\left(\mathrm{G}, \mathrm{H}_{1}, \mathrm{E}\right) / \mathrm{P}(\mathrm{G}, \mathrm{E}) \\
& \mathrm{P}\left(\mathrm{H}_{1} / \mathrm{G}, \mathrm{E}\right)=\mathrm{P}\left(\mathrm{G}, \mathrm{H}_{1}, \mathrm{E}\right) / \mathrm{P}(\mathrm{G}, \mathrm{E}) \\
& \mathrm{P}\left(\mathrm{H}_{1} / \mathrm{G}, \mathrm{E}\right)=\mathrm{P}\left(\mathrm{G}_{1} / \mathrm{H}_{1}, \mathrm{E}\right) * \mathrm{P}\left(\mathrm{H}_{1} / \mathrm{E}\right) / \mathrm{P}(\mathrm{G} / \mathrm{E}) \\
& \text { POSTERIOR ODDS }= \text { PRIOR PROBABILITY } \\
& \times \text { LIKELIHOOD RATIO }
\end{aligned}
$$

Exhalations of radon in the soil-air and in groundwater only define the region where the strain is likely to exist and where changes may occur in faults, cracks and active tectonic blocks [24]. Thus posterior probability of occurrence of deformation strain with radon anomaly increase under pore pressure increase depends on the likelihood of occurrence of deformation strain and radon anomaly fluctuation with pore pressure. So for a homogenous model if
$\mathrm{P}\left(\mathrm{H}_{1} / \mathrm{E}\right)$ is the conditional probability that strain increases with increasing pore pressure where $\mathrm{P}(\mathrm{G} / \mathrm{E})$ is the conditional probability of radon anomaly increase. We can say that as the probability of strain increase with radon anomaly increase and the pore pressure increases the probability of radon anomaly with pore pressure rise will slowly decrease. From this equation it is clear that the radon anomaly increase under the condition that strain increases with increasing pore pressure and also that strain increase is due to pore pressure change. Discharge of strain occurs in non elastic process and aftershock sequence with accumulation of strain energy occurs due to slow quasi elastic increase of pressure within source. Elastic breakdown of material cause discharge of strain energy. Thus at the probability of maximum strain condition with increasing radon with pore pressure; the radon anomaly with increasing pore pressure will be minimum. Here it is proved that at the maximum deformation condition the radon anomaly will be the minimum and will decline. A bayesian framework with background pore pressure thus explains the condition of maximum strain change with radon anomaly. The above bayesian framework confirms through a probabilistic model how the system behaves when the strain value is maximum and decides how radon anomaly change decreases before undergoing complete fracture of the rock prior to the system undergoing maximum strain with background evidence of porosity. We also derive an optimistic bayesian outlook of the slip predicttive nature of a block for a distribution and find out the expectation of a radon anomaly change with the evidence that pore pressure increases for the block. If lithospherecal block is not in a critical condition on a pressure any trigger's function cannot cause earthquake. Nucleation of an earthquake in neighbor fault blocks causes no influence on the radon behavior. If a lithospheric block has new pore formation and the nature of radon anomaly drop is known, a probabilistic analysis can be made of the strain deformation. The unknown number of files having the same characteristics that is slip patching in the same procedure possessing characteristic $\mathrm{x}$ be denoted by M. Before obtaining any evidence, we can take $M$ to have the binomial distribution $\operatorname{Bin}(\mathrm{N}+1 ; \mathrm{P})$. Now we have observed that $\mathrm{S}$ has characteristic $\mathrm{x}$, and so have learned that $M \geq 1$. If $M=1$ there is no other matching lithospheric block, and $\mathrm{S}$ must be only source; however, if there is a non-negligible probability that $\mathrm{M}>1$, so that $\mathrm{S}$ is not the only matching block, this would be a source of doubt as to S's condition of nucleation.

$$
\begin{aligned}
\operatorname{Pr}(\mathrm{M}>1 \mid \mathrm{M}<=1)= & 1-(1-\mathrm{P})^{\mathrm{N}+1} \\
& -(\mathrm{N}+1) \mathrm{P}(1-\mathrm{P})^{\mathrm{N}} / 1-(1-\mathrm{P})^{\mathrm{N}+1}
\end{aligned}
$$

A Bayesian melding framework draws inference that there is only a single file or lithospheric block which ex- 
perience radon anomaly $(\mathrm{G})$ and break undergoes slip nucleation then $\operatorname{Pr}(\mathrm{G} \mid \mathrm{M}=\mathrm{m})=\mathrm{m}^{-1}$. As above, we condition the initial $\operatorname{Bin}(\mathrm{N}+1 ; \mathrm{P})$ for $\mathrm{M}$ on the known fact that $\mathrm{M} \geq 1$, to obtain:

$$
\operatorname{Pr}(\mathrm{G} \mid \mathrm{E})=\mathrm{E}\left(\mathrm{M}^{-1} \mid \mathrm{M} \geq 1\right)
$$

Let us consider given the evidence, we know that there is one which has a file slipping and out of the remaining $\mathrm{N}$ blocks, each has, independent, probability $\mathrm{P}$ of supplying a slip patch condition. So the conditional distribution of $\mathrm{M}$ is $1+\mathrm{Bin}(\mathrm{N}$; $\mathrm{P})$. Using this to take the expectation of $\mathrm{M}^{-1}$ yields

$$
\operatorname{Pr}(\mathrm{G} \mid \mathrm{E})=1-(1-\mathrm{P})^{\mathrm{N}+1} /(\mathrm{N}+1) \mathrm{P}
$$

In our case study, the posterior distribution of the strain deformation in a collection of zones is that of a sum of random variables, each of which has a distribution that is a mixture of several truncated normal components. Although this is complicated to find analytically, it is easy to evaluate by simulation (Figure 3).

\section{Results and Discussion}

Seismic precursory model of radon gas emanation in the process of earthquake prediction research finds out the distinct anomaly variation necessary to correlate radon gas with processes of preparation and realization of tectonic earthquakes in long-term and short-term forecasts tectonic earthquakes. We conclude that Radon gas can be validated as an earthquake precursor though an acceptable geodynamic modeling and accounting for its occurrence is a challenging task. Geodynamic monitoring provides a host of challenging situation and is advantageous for radon detector placed directly in the researched block of rocks for the signal of radon monitoring that permits stress dynamics in intense deformed conditions of the block of rocks due to compression or a stretching. Due to

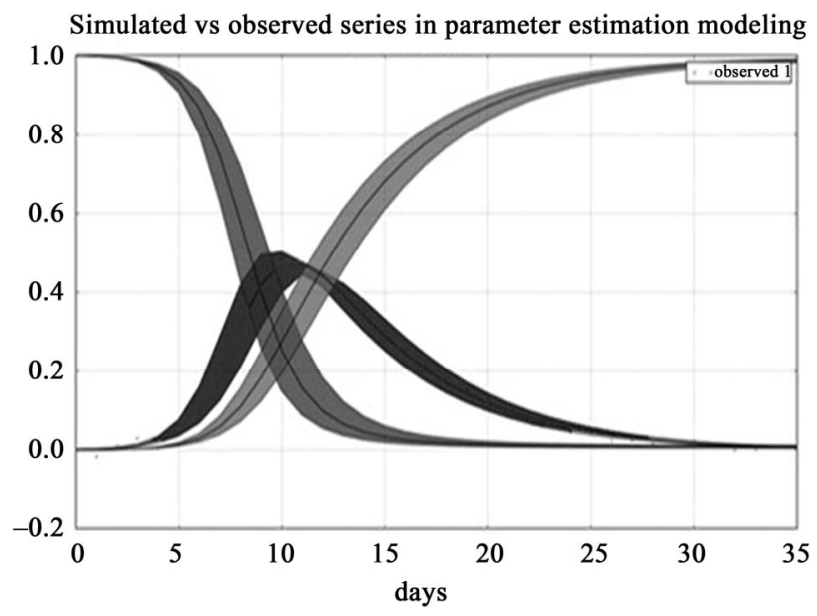

Figure 3. Parameter estimation in dynamic model for radon volume analysis. such a condition speed of processes of dynamics (changes) of rocks by preparation of earthquake is rather insignificant but offers host of processing probabilistic or melding frameworks for monitoring in a mode of real time. Bayesian melding was applied to deterministic simulation model in which information from prior knowledge and a deterministic computer model is conditioned on a likelyhood function. Our study involves extended Bayesian melding to deal with stochastic simulation models for strain condition based on radon anomaly changes occurring in the geodynamic model. Bayesian melding combines all the available information about model inputs and outputs and combines them in a bayesian way, to provide a posterior distribution of quantities of interest that provides a fully assessment of uncertainty and risk. It would then be possible to model spatial correlation by replacing the independence assumption by a joint distribution with dependence specified by a geo-statistical correlation function. Something like this was done for probabilistic weather forecasting by [25] and is found to permit meso-micro scale integration through use of simulated data. Something like this was done for probabilistic weather forecasting by [26] and is found to permit meso-micro scale integration through use of simulated data. This method needs to be enhanced further with sophisticated descriptive and predictive models of formation and transformation of other seismo-geochemical precursor signals, implementing algorithms to extract useful information from field data, selecting the criteria for the best sensitivity of radon based precursors to the earthquakes and optimizing observation networks.

This analytical approach may be used in assessment of radon anomalous pattern in the seismogenic regions of India (Peninsular and Extra-peninsular) and other analogous tectonics of the world to understand earthquake precursory behavior where occurrence of tectonic earthquakes are very much prevalent due to in-situ material heterogeneities having enough propensity to emanate radon gas $[27,28]$. The present methodology incorporates a deterministic simulation model for parameter estimation for one distribution to produce a sample from a different distribution not incorporated earlier in [29].

\section{REFERENCES}

[1] H. Wakita, "Geochemical Challenge to Earthquake Prediction," Proceedings of the National Academy of Sciences, Vol. 93, No. 9, 1996, pp. 3781-3786.

[2] V. Walia, "Earthquake Prediction Studies Using Radon as a Precursor in N-W Himalayas, India: A Case Study," TAO, Vol. 16, No. 4, 2005, pp. 775-804.

[3] V. I. Ulomov and B. Z. Mavashev, "On Forerunner of a Strong Tectonic Earthquake," Doklady Akademii nauk SSSR, Vol. 176, 1967, pp. 319-322.

[4] I. Miklavcić, V. Radolić, B. Vuković, M. Poje, M. Varga, 
D. Stanić and J. Planinić, "Radon Anomaly in Soil Gas as an Earthquake Precursor," Applied Radiation and Isotopes, Vol. 66, No. 10, 2008, pp. 1459-1466.

[5] T. Teng, "Some Recent Studies on Ground Water Random Content as an Earthquake Precursor," Journal of Geophysical Research, Vol. 85, No. B6, 1980, pp. 30893099. doi:10.1029/JB085iB06p03089

[6] H. H. Shapiro, J. D. Melvin, T. A. Tombrello, H. H. Mendenhall, P. B. Larson and J. H. Whitcomb, "Relationship of the 1979 Southern California Radon Anomaly to a Possible Regional Strain Event," Journal of Geophysical Research, Vol. 86, No. B3, 1981, pp. 1725-1730.

[7] I. P. Dobrovosky, S. A. Zubkov and V. I. Miachkin, "Estimation of the Size of the Earthquake Preparation Zones," Pure and Applied Geophysics, Vol. 117, No. 5, 1979, pp. 1025-1044. doi:10.1007/BF00876083

[8] E. Hauksson, "Radon Content of Ground Water as an Earthquake Precursor: Evaluation of the World Wide Data and Physical Basis," Journal of Geophysical Research, Vol. 86, No. B10, 1981, pp. 9397-9410.

[9] C. Scholz, "Earthquake Prediction: A Physical Basis Science," Science, Vol. 181, No. 4102, 1973, pp. 803-810. doi:10.1126/science.181.4102.80310

[10] B. T. Brady, "Anomalous Seismicity Prior to Rock Bursts: Implications for Earthquake Prediction," Pure and Applied Geophysics, Vol. 115, No. 1-2, 1991, pp. 357-374. doi:10.1007/BF01637114

[11] V. I. Utkin and A. K. Yurkov, "Radon as a Tracer of Tectonic Movements," Russian Geology and Geophysics, Vol. 51, No. 2, 2010, pp. 220-227. doi:10.1016/j.rgg.2009.12.022

[12] D. Poole and A. E. Raftery, "Inference for Deterministic Simulation Models: The Bayesian Melding Approach," Journal of the American Statistical Association, Vol. 95, No. 452, 2000, pp. 1244-1255. doi:10.2307/2669764

[13] R. C. Ramola, Y. Prasad, G. Prasad, S. Kumar and V. M. Choubey, "Soil-Gas Radon as Seismotectonic Indicator in Garhwal Himalaya," Applied Radiation and Isotopes, Vol. 66, No. 10, 2008, pp. 1523-1530.

[14] V. G. Bakhmutov and A. A. Groza, "Dilatancy Difussion Model: New Prospects," Proceedings of the 7th International Conference "Problems of Geocosmos", St. Petersburg, 26-30 May 2008, pp. 3-4.

[15] G. I. Voitov, A. S. Gusev, N. S. Kozlova, V. P. Rudakova and V. N. Shuleikin, "Emanation and Electrical Effects above Complex Tectonic Structures (Aleksandrovskaya Zone of Fault-Line Uplifts, Belorussia)," Doklady Akademii Nauk, Vol. 370, No. 1, 2000, pp. 105-108.

[16] P. P. Firstov and V. P. Rudakov, "Results of Recording of Subsurface Radon in 1997-2000 at the Petropavlovsk Kamchatski Geodynamic Research Area," Vulkanologiya i Seismologiya, Vol. 1, 2003, pp. 26-41.

[17] C.-Y. King and Y. Chi, "Gas-Geochemical Approaches to Earthquake Prediction. Isotopic and Geochemical Precursors of Earthquakes and Volcanic Eruptions," Proceedings of an Advisory Group Meeting, Vienna, 9-12
September 1991, pp. 9-12.

[18] R. F. Holub and B. T. Brady, "The Effect of Stress on Radon Emanation from Rock," Journal of Geophysical Research, Vol. 86, No. B3, 1981, pp. 1776-1784. doi:10.1029/JB086iB03p01776

[19] K. Katoh, O. Nishizawa, K. Kuronose and K. Ikeda, “An Experimental Study on Variation of Radon Emanation from Westerly Granite under Uniaxial Compression Part 1," Journal of the Seismological Society of Japan, Vol. 38, 1985, pp. 173-182.

[20] M. Trique, P. Richon, F. Perrier, J. P. Avouac and J. C. Sobroux, "Radon Emanation and Electric Potential Variation Associated with Transient Deformation Near Reservoir Lakes," Nature, Vol. 399, 1991, pp. 137-141. doi:10.1038/20161

[21] V. I. Utkin, E. Mamyrov, M. V. Kan, S. V. Krivasheev, A. K. Yurkov, I. I. Kosyakin and A. N. Shishkanov, "Radon Monitoring in the Northern Tien Shan with Application to the Process of Tectonic Earthquake Nucleation," Izvestiya Physics of the Solid Earth, Vol. 42, No. 9, 2006, pp. 775784.

[22] Y. Kawada, H. Nagahama, Y. Omori, Y. Yasuoka, T. Ishikawa, S. Tokonami and M. Shinogi, "Time-Scale Invariant Changes in Atmospheric Radon Concentration and Crustal Strain Prior to a Large Earthquake," Nonlinear Processes in Geophysics Vol. 14, 2007, pp. 123-130. doi:10.5194/npg-14-123-2007

[23] M. Snnirman and E. Blanter, "Hierarchial Model of Seismicity," Non Linear Dynamics of Lithosphere and Earthquake Prediction, Springer, 2008, pp. 40-41.

[24] A. Gelman and D. B. Rubin, "Inference from Iterative Simulation Using Multiple Sequences," Statistical Science, Vol. 7, No. 4, 1992, pp. 457-472. doi:10.1214/ss/1177011136

[25] V. T. Dubinchuk, "Radon as a Precursor of Earthquakes; Isotopic and Geochemical Precursors of Earthquakes and Volcanic Eruptions," IAEA-TECDOC-726, 1993, p. 7.

[26] Y. Gel, A. E. Raftery and T. Gneiting, "Calibrated Probabilistic Mesoscale Weather Field Forecasting: The Geostatistical Output Perturbation (GOP) Method (with Discussion)," Journal of the American Statistical Association, Vol. 99, No. 467, 2004, pp. 575-583. doi: $10.1198 / 016214504000000872$

[27] O. P. Mishra, "Lithospheric Heterogeneity and Seismotectonics of NE Japan Forearc and Indian Regions," unpublished D.Sc. Thesis, Ehime University, Japan, 2004, p. 223.

[28] S. Mukhopadhyay, O. P. Mishra, D. Zhao and J. Kayalal, "2006: 3-D Seismic Structure of the Source Area of the 1993 Latur, India Earthquake and Its Implications for Rupture Nucleations," Tectonophysics, Vol. 415, pp. 1-16.

[29] P. K. Dutta, M. K. Naskar and O. P. Mishra, "Test of Strain Behavior Model with Radon Anomaly in Earthquake Prone Zones," Himalayan Geology, Vol. 33, No. 1, 2012, pp. 23-28. 